PROCEEDINGS OF THE

AMERICAN MATHEMATICAL SOCIETY

Volume 129, Number 1, Pages 303-309

S 0002-9939(00)05812-3

Article electronically published on August 30, 2000

\title{
THERE EXISTS A POLYHEDRON WITH INFINITELY MANY LEFT NEIGHBORS
}

\author{
DANUTA KOŁODZIEJCZYK
}

(Communicated by Ralph Cohen)

\begin{abstract}
We show that there exists a finite polyhedron $P$ homotopy dominating infinitely many finite polyhedra $K_{i}$ of different homotopy types such that there isn't any homotopy type between $P$ and $K_{i}$. This answers negatively the question raised by K. Borsuk in 1975: Does every FANR have only finitely many left neighbors?
\end{abstract}

We assume the reader to be familiar with the basic notions of shape theory. The classical works here are [B2], [DS], [MS].

Following K. Borsuk ([B2], p. 349) we call a shape $\operatorname{Sh}(X)$ a left neighbor of $\operatorname{Sh}(Y)$ iff $X$ is shape dominated by $Y, Y$ is not shape dominated by $X$, and every $Z$ such that $X$ is shape dominated by $Z$ and $Z$ is shape dominated by $Y$ has the shape of either $X$ or $Y$ (in the same manner we define a right neighbor of $\operatorname{Sh}(Y)$ ).

Concerning this notion K. Borsuk asked in his monograph in 1975 ([B2], Problem (6.4), p. 349):

Is it true that every FANR has only a finite number of left neighbors?

We answer this question showing that there exists even a finite polyhedron with infinitely many left neighbors which are also finite polyhedra.

Remark 1. Similarly, in the homotopy category of compact ANR's there were defined the so-called $h$-neighbors (see [B1]). Since on this category shape theory and homotopy theory coincide, we immediately obtain an example of a finite polyhedron with infinitely many left $h$-neighbors.

Let us note that from the results of Hastings and Heller ([HaHe1], [HaHe2]), there is a $1-1$ correspondence between the shapes of compacta and the homotopy types of $C W$-complexes dominated by a given polyhedron.

Hence we will work in the homotopy category of $C W$-complexes and homotopy classes of maps between them.

Remark 2. On the other side, it is easy to find a finite polyhedron with infinitely many right neighbors. It suffices to take a point $\{p\}$, then the spheres $S^{i}$, for

Received by the editors February 28, 1999.

2000 Mathematics Subject Classification. Primary 55P55, 55P15.

Key words and phrases. Shape, homotopy type, FANR, polyhedron, shape domination, homotopy domination, left neighbor.

The author would like to thank the Institute of Mathematics of the Polish Academy of Sciences for its support while this work was done. 
$i=1,2 \ldots$, are the right neighbors of $\{p\}$ (by the above-mentioned results of Hastings and Heller and the classical Whitehead Theorem, this claim is obvious).

Definition 1. Let $\pi$ be a group and $m$ an integer $\geq 2$. A $(\pi, m)$-complex $X$ is a finite, connected $C W$-complex with dimension $\leq m$ having fundamental group $\pi_{1}(X)$ isomorphic to $\pi$ and trivial homotopy modules $\pi_{i}(X)$ for $1<i<m$.

In 1949 J. H. C. Whitehead proved the following:

Theorem (Theorem 14, Wh2]; see also [Wh1]). Assume that $X$ and $Y$ are $(\pi, m)$ complexes. Then there exist integers $a_{X}$ and $a_{Y}$ such that

$$
X \vee \bigvee_{a_{X}} S^{m} \simeq Y \vee \bigvee_{a_{Y}} S^{m}
$$

where $\bigvee_{k} S^{m}$ denotes the wedge of $k$ spheres $S^{m}$.

Thus the homotopy types of $(\pi, m)$-complexes form a connected tree, in which the homotopy type of $X$ is joined by an edge to the homotopy type of $X \vee S^{m}$ (this graph clearly contains no circuits). We denote it by $\mathrm{HT}(\pi, m)$.

Each tree is partitioned into levels by the Euler-Poincaré characteristic $\chi$, and note that if $\chi(X)=\chi(Y)$, then $a_{X}=a_{Y}$ in the above theorem. By junctions in a tree we mean the homotopy types with two or more inequivalent factorizations involving an $S^{m}$ summand.

Our example which answers the question of K. Borsuk will be an infinite junction in some 3-dimensional tree.

Definition 2. A finitely generated projective $\mathbf{Z} \pi$-module $M$ is called stable free iff there exist finitely generated free $\mathbf{Z} \pi$-modules $(\mathbf{Z} \pi)^{(r)}$ and $(\mathbf{Z} \pi)^{(s)}$ (where $r$ and $s$ are integers) such that

$$
M \oplus(\mathbf{Z} \pi)^{(r)} \cong(\mathbf{Z} \pi)^{(s)}
$$

$\left((\mathbf{Z} \pi)^{(n)}\right.$ denotes the direct sum of $n$ copies of $\left.\mathbf{Z} \pi\right)$.

By this definition, if $M_{1}$ and $M_{2}$ are two finitely generated projective $\mathbf{Z} \pi$-modules which are stable free, then

$$
M_{1} \oplus(\mathbf{Z} \pi)^{\left(r_{1}\right)} \cong M_{2} \oplus(\mathbf{Z} \pi)^{\left(r_{2}\right)},
$$

for some integers $r_{1}$ and $r_{2}$. Thus one can consider the tree of such modules as the tree of homotopy types.

In the sequel we will use the following lemma (compare [Dy2], p. 253; for the sketch of the proof, see the Appendix).

Lemma. If there exists a finite $C W$-complex $K(\pi, 1)$ of dimension $n$, then the tree $\mathrm{HT}(\pi, m)$, for $m \geq \max (n, 3)$, is identical with the tree of isomorphism classes of stable free, finitely generated projective $\mathbf{Z} \pi$-modules.

Definition 3. Recall that a group $G$ is a poly-Z-group if it has a finite normal series

$$
G=G_{0} \supseteq G_{1} \supseteq \ldots \supseteq G_{n}=1
$$

$\left(G_{i} \triangleleft G, G_{i} \triangleleft G_{i-1}\right.$, for $\left.i=1, \ldots, n\right)$ with factors $G_{i-1} / G_{i} \cong \mathbf{Z}$.

The number $h(G)$ of the terms $\mathbf{Z}$ in a decomposition as above is an invariant of $G$ known as the Hirsch number of $G$ (see $\underline{\mathbb{R}}$, (5.4.13)). 
Definition 4. A group $G$ is called Hopfian if every epimorphism $f: G \rightarrow G$ is an automorphism.

Definition 5. A group $G$ is residually finite iff all its subgroups of finite index have trivial intersection.

Let us now prove the following theorem.

Theorem. There exists a finite polyhedron $P$ with infinitely many left neighbors.

Proof. Let $G=T / T^{(2)}$, where $T$ is the trefoil knot group, i.e. the group with a presentation

$$
T=\left\langle a, b \mid a^{2}=b^{3}\right\rangle
$$

$\left(T^{(2)}\right.$ denotes the second derived of the group $T$, i.e. $T^{(2)}=\left(T^{\prime}\right)^{\prime}$, where, as usual, $\left.T^{\prime}=[T, T]\right)$. Then we have $G^{\prime} \cong \mathbf{Z} \oplus \mathbf{Z}$ and the sequence

$$
1 \triangleleft \mathbf{Z} \oplus \mathbf{Z} \cong G^{\prime} \triangleleft G,
$$

in which $G / G^{\prime} \cong \mathbf{Z}$ (moreover, it is easily seen that $G^{(2)}=1$ ).

Thus $G$ is a poly-Z-group with a Hirsch number $h(G)=3$ (and a metabelian group); hence there exists a finite $\mathrm{CW}$-complex $\mathrm{K}(\mathrm{G}, 1)$ of dimension $\leq 3$ (see for example Theorem 7.1, Ch. 8, $[\mathrm{Br}]$ ).

P. Berridge and M. Dunwoody showed in $[\mathrm{BeDu}]$ that there exist infinitely many non-isomorphic projective modules $M_{i}$ over $\mathbf{Z} G$ (of rank 1). Moreover $M_{i} \oplus \mathbf{Z} G \cong$ $\mathbf{Z} G \oplus \mathbf{Z} G$ (see $[\mathrm{BeDu}]$ ), so these modules are projective and stable free, and obviously, finitely generated.

Thus, by the Lemma, there is an infinite junction in the homotopy tree $\operatorname{HT}(G, 3)$, i.e. there exist infinitely many finite polyhedra $K_{i}$ of different homotopy types and a polyhedron $P$ such that $K_{i} \vee S^{3} \simeq P$ with $\pi_{1}\left(K_{i}\right) \cong \pi_{1}(P) \cong G, \pi_{2}\left(K_{i}\right) \cong$ $\pi_{2}(P)=0$ and $\operatorname{dim} K_{i}, \operatorname{dim} P \leq 3$.

We will show that $K_{i}$ are left neighbors of $P$.

Consider any $X \leq P$ (where $X \leq P$ denotes that $X$ is homotopy dominated by $P)$.

By the results of Wall if $X$ is a $C W$-complex dominated by a finite polyhedron $P$ with $\operatorname{dim} P=n \geq 2$ and $\tilde{K}^{0}\left(\mathbf{Z} \pi_{1}(X)\right)=0$ (for the definition of the group $\tilde{K}^{0}(\mathbf{Z} G$ ), see $\mathrm{Wa}$ ), then $X$ is homotopy equivalent to a finite polyhedron of dimension $\leq$ $\max (n, 3)(\mathrm{Wa}$, Theorem F, p. 66).

In our situation $\tilde{K}^{0}\left(\mathbf{Z} \pi_{1}(X)\right)=0$ because from the results of Waldhaussen $([\mathrm{Wd}]$ ), this is true for every poly-Z-group and all of its subgroups (obviously each subgroup of a poly-Z-group is a poly-Z-group). Thus we may assume every $X \leq P$, where $P$ is as above, to be a finite polyhedron of dimension $\leq 3$.

Let $K$ denote any $K_{i}$.

Suppose that there exists an $L$ such that $K \leq L \leq P$. Let us then observe that $\pi_{1}(L) \cong G$. Otherwise, there exists an $r$-homomorphism $G \cong \pi_{1}(P) \rightarrow \pi_{1}(K) \cong G$ that is not an isomorphism, which is impossible.

Indeed, it was proven by Hirsch that every polycyclic group is residually finite (see (5.4.17), $[\mathrm{R}$ and by Malcev that if a finitely generated group is residually finite, then it is Hopfian (see [Ma], $[\mathrm{KS}]$ ).

Therefore $L$ belongs to our tree.

Since $K \leq L \leq P$ and $K \vee S^{3} \simeq P$, then either $\chi(L)=\chi(K)$ or $\chi(L)=\chi(P)$. 
Consider the case where $\chi(L)=\chi(K)$. It follows from the Whitehead Theorem on Trees that

$$
K \vee \bigvee_{a} S^{3} \simeq L \vee \bigvee_{a} S^{3}
$$

for some integer $a$.

For any $(\pi, m)$-complex $X, \pi_{m}\left(X \vee S^{m}\right) \cong \pi_{m}(X) \oplus \pi_{m}\left(S^{m}\right)$ as $\mathbf{Z} \pi$-modules (folklore). Then we have

$$
\pi_{3}(K) \oplus(\mathbf{Z} G)^{(a)} \cong \pi_{3}(L) \oplus(\mathbf{Z} G)^{(a)}
$$

as $\mathbf{Z} G$-modules. Hence, for a sufficiently large integer $b$, there is

$$
\pi_{3}(K) \oplus(\mathbf{Z} G)^{(b)} \cong \pi_{3}(L) \oplus(\mathbf{Z} G)^{(b)} \cong(\mathbf{Z} G)^{(d)},
$$

where $d$ is an integer (recall that there exists a $K(G, 1)$ at this tree and use the Theorem of Whitehead on Trees once again).

Since $K \leq L$, if the homotopy type of $L$ is different from that of $K$, then there exists a nontrivial $\mathbf{Z} G$-module $C$ such that $\pi_{3}(K) \oplus C \cong \pi_{3}(L)$. Indeed, as above - since $G$ is Hopfian, the domination of $L$ over $K$ induces an isomorphism of fundamental groups. If it also induces an isomorphism of $\pi_{3}$, then by the classical Whitehead Theorem, $K$ and $L$ are homotopy equivalent (recall that $\left.\pi_{2}(K)=\pi_{2}(L)=0\right)$.

Then we have $(\mathbf{Z} G)^{(d)} \oplus C \cong(\mathbf{Z} G)^{(d)}$; hence $(\mathbf{Z} G)^{(d)}$ is isomorphic to a proper direct factor of itself. But this is impossible because for any group $G$, any finitely generated free $\mathbf{Z} G$-module $(\mathbf{Z} G)^{(r)}$ (where $r$ is an integer) cannot be isomorphic to a proper direct factor of itself - this is a corollary to the result of Kaplansky ( $\mathrm{Ka}$, p. 122 ; see $[\mathrm{Pa}]$ ).

By the same arguments $P$ cannot dominate $L$ of the homotopy type different from that of $P$ in the case where $\chi(L)=\chi(P)$.

Obviously, $K$ cannot dominate $P$. Thus $K$ is a left neighbor of $P$ and the proof is finished.

Remark 3. The group $G$ in consideration has the following presentation (see [BeDu] $)$

$$
G=\left\langle r, p, q \mid p q=q p, q=r^{-1} p r, r^{-1} q r=q p^{-1}, r p r^{-1}=q^{-1} p, r q r^{-1}=p\right\rangle .
$$

\section{APPENDIX}

The Lemma can be drawn from the classical results of J. H. C. Whitehead and S. Mac Lane and constructions similar to those of Wall (compare the proof of Theorem 7.1, Ch. 8 in $[\mathrm{Br}$ and the proof of Proposition 1.4 in [Dy1]).

First recall some definitions (see [MWh, Dy1]):

Definition 6. By an algebraic m-type we mean a triple $T=\left(\pi, \pi_{m}, k\right)$, where $\pi$ is a group, $\pi_{m}$ is a $\mathbf{Z} \pi$-module, and $k \in H^{m+1}\left(\pi, \pi_{m}\right)$.

Definition 7. The algebraic $m$-type of a given $C W$-complex $X$ whose universal cover $\tilde{X}$ is $(m-1)$-connected is the triple

$$
T(X)=\left(\pi_{1}(X), \pi_{m}(X), k(X)\right),
$$

where $k(X) \in H^{m+1}\left(\pi_{1}(X), \pi_{m}(X)\right)$ is the first $k$-invariant of $X$ (for its definition, see [MWh]). 
Definition 8. We call two algebraic $m$-types $T=\left(\pi, \pi_{m}, k\right)$ and $T^{\prime}=\left(\pi, \pi_{m}^{\prime}, k^{\prime}\right)$ isomorphic iff there exists an automorphism $\theta: \pi \rightarrow \pi$, and a $\theta$-automorphism $\beta$ : $\pi_{m} \rightarrow \pi_{m}^{\prime}$ (i.e. $\beta(\alpha \cdot y)=\theta(\alpha) \beta(y)$, for $\left.\alpha \in \pi, y \in \pi_{m}\right)$ such that $k=\left(\beta^{*}\right)^{-1} \cdot \theta^{*}\left(k^{\prime}\right)$ in the diagram

$$
H^{m+1}\left(\pi ; \pi_{m}\right) \stackrel{\beta^{*}}{\rightarrow} H^{m+1}\left(\pi ;\left(\pi_{m}^{\prime}\right)_{\theta}\right) \stackrel{\theta^{*}}{\leftarrow} H^{m+1}\left(\pi ; \pi_{m}^{\prime}\right),
$$

where $\left(\pi_{m}^{\prime}\right)_{\theta}$ is the $\pi$-module with action $\alpha * y=\theta(\alpha) \cdot y$, for $\alpha \in \pi, y \in \pi_{m}^{\prime}$.

Definition 9. Two connected $C W$-complexes $X$ and $Y$ have the same homotopy $m$-type if there exists a map $f: X^{(m+1)} \rightarrow Y^{(m+1)}$ such that $f_{i *}: \pi_{i}\left(X^{(m+1)}\right) \rightarrow$ $\pi_{i}\left(Y^{(m+1)}\right)$ is an isomorphism for all $1 \leq i \leq m$.

It is known that two $C W$-complexes $X$ and $Y$ such that $\tilde{X}$ and $\tilde{Y}$ are $(m-$ 1 )-connected have the same homotopy $m$-type iff $T(X) \cong T(Y)$. In particular, the homotopy type of a $(\pi, m)$-complex $X$ is completely determined by $T(X)$ (see [MWh] $)$.

Sketch of the proof of the Lemma. Let us take $m \geq \max (n, 3)$ and consider the tree of $(\pi, m)$-complexes. Observe that $\pi_{m}(X)$ of each $(\pi, m)$-complex $X$ is a finitely generated, projective, stable free $\mathbf{Z} \pi$-module (since there exists a $(\pi, m)$-complex $K(\pi, 1)$, it follows from the Whitehead Theorem on Trees).

On the other hand, let $\pi_{m}$ be a finitely generated, projective, stable free $\mathbf{Z} \pi$ module. It was shown in [Wh1, II that every abstract $m$-type $T=\left(\pi, \pi_{m}, k\right)$ can be realized by a connected $(m+1)$-dimensional CW-complex $X$ (not necessarily finite) such that $T(X) \cong T$. Moreover, if there exists a finite $C W$-complex $K(\pi, 1)$ of dimension $\leq n, X$ can be choosen to be finite (the construction may be done by the methods of homological algebra similarly to the proof of Theorem 7.1, Ch. 8 in $\mathrm{Br}]$ ).

Let us observe that for such an $X$ we have $\pi_{m}(X) \cong C_{m}(\tilde{X}) / B_{m}(\tilde{X})$, where $C_{m}(\tilde{X})$ are the $m$-th chains in the cellular chain complex of the universal cover $\tilde{X}$ of $X$

$$
\mathcal{C}: C_{m+1}(\tilde{X}) \stackrel{\partial_{m+1}}{\longrightarrow} C_{m}(\tilde{X}) \stackrel{\partial_{m}}{\longrightarrow} C_{m-1}(\tilde{X}) \stackrel{\partial_{m-1}}{\longrightarrow} C_{m-2}(\tilde{X}) \stackrel{\partial_{m-2}}{\longrightarrow} \ldots \stackrel{\partial_{1}}{\longrightarrow} C_{0}(\tilde{X})
$$

and $B_{m}(\tilde{X})=\operatorname{im} \partial_{m+1}$.

By the assumption, $\pi_{m} \cong \pi_{m}(X)$ is a projective, stable free, finitely generated module over $\mathbf{Z} \pi$. Thus we have

$$
C_{m}(\tilde{X}) / B_{m}(\tilde{X}) \oplus(\mathbf{Z} \pi)^{(s)} \cong(\mathbf{Z} \pi)^{(t)},
$$

for some integers $s$ and $t$.

In this situation

$$
\mathcal{C}^{\prime}: C_{m}(\tilde{X}) / B_{m}(\tilde{X}) \oplus(\mathbf{Z} \pi)^{(s)} \stackrel{\partial_{m}^{\prime}}{\longrightarrow} C_{m-1}(\tilde{X}) \oplus(\mathbf{Z} \pi)^{(s)} \stackrel{\partial_{m-1}^{\prime}}{\longrightarrow} C_{m-2}(\tilde{X}) \stackrel{\partial_{m-2}^{\prime}}{\longrightarrow} \ldots
$$

with $\partial_{m}^{\prime}=\partial_{m} \oplus i d, \partial_{m-1}^{\prime}=\partial_{m-1} \oplus 0$ and $\partial_{i}^{\prime}=\partial_{i}$, for $i \leq m-2$ is a cellular chain complex of free, finitely generated $\mathbf{Z} \pi$-modules.

Let us take an $m$-dimensional $C W$-complex

$$
X^{\prime}=\left(X^{(m-1)} \vee \bigvee_{s} S^{m-1}\right) \cup \bigcup_{i=1}^{t} e_{i}^{m},
$$


where the $m$-cells are attached to $X^{(m-1)} \vee \bigvee_{s} S^{m-1}$ by the maps corresponding to the

$$
\partial_{m}^{\prime}:(\mathbf{Z} \pi)^{(t)} \rightarrow C_{m-1}(\tilde{X}) \oplus(\mathbf{Z} \pi)^{(s)}
$$

(compare the proof of Proposition 1.4 in Dy1]).

Since $\pi_{m}\left(X^{\prime}\right) \cong H_{m}(\tilde{X})=\operatorname{ker} \partial_{m}^{\prime} / \mathrm{im} \partial_{m+1}^{\prime}$, then it is easily seen that $\pi_{m}\left(X^{\prime}\right) \cong$ $\pi_{m}(X)$.

Moreover, it is clear that there is a 1-1 correspondence between isomorphism classes of finitely generated, projective and stable free $\mathbf{Z} \pi$-modules and the homotopy types of $(\pi, m)$-complexes (note that in our situation $H^{m+1}\left(\pi_{1}(X), \pi_{m}(X)\right)=$ $0)$.

Remark 4. The proof of the main Theorem may now be slightly simplified in the last part. Indeed, by the proof of the Lemma, two $(G, m)$-complexes with distinct homotopy types have non-isomorphic groups $\pi_{m}$; hence the existence of a $\mathbf{Z} G$ module $C \neq 0$ such that $\pi_{3}(K) \oplus C \cong \pi_{3}(L)$ is immediate.

\section{ACKNOWLEDGMENTS}

The author would like to thank Professor Jerzy Dydak for valuable remarks concerning the manuscript.

\section{REFERENCES}

[BeDu] P. H. Berridge, M. J. Dunwoody, Non free projective modules for torsion-free groups, J. London Math. Soc. (2) 19 (1979), 433-436. MR 80k:20041

[B1] K. Borsuk, Theory of Retracts, Polish Scientific Publishers 44, Warsaw, 1967. MR 35:7306

[B2] K. Borsuk, Theory of Shape, Polish Scientific Publishers 59, Warsaw, 1975. MR 54:6132

[Br] K. Brown, Cohomology of Groups, Springer, Berlin, 1982. MR 83k:20002

[DS] J. Dydak, J. Segal, Shape Theory: An Introduction, Lecture Notes in Math. 688, Springer, Berlin, 1978. MR 80h:54020

[Dy1] M. N. Dyer, Homotopy classification of $(\pi, m)$-complexes, J. Pure Appl. Alg. 7 (1976), 249-282. MR 58:2798

[Dy2] M. N. Dyer, Trees of homotopy types of $(\pi, m)$-complexes, Homological Group Theory, London Math. Soc. Lecture Notes 36, 1979, 251-254. MR 81f:55003

[HaHe1] H. M. Hastings, A. Heller Homotopy idempotents on finite-dimensional complexes split, Proc. Amer. Math. Soc. 85 (1982), 4, 619-622. MR 83j:55010

[HaHe2] H. M. Hastings, A. Heller, Splitting homotopy idempotents, Shape Theory and Geom. Top. Proc. (Dubrovnik, 1981), Lecture Notes in Math. 870, Springer, Berlin, 1981, 2536. MR 83a:55017

[Ka] I. Kaplansky, Fields and Rings, University of Chicago Press, 1972. MR 50:2139

[KS] A. Karras, D. Solitar, On free products, Proc. Amer. Math. Soc. 9, (1958), 217-221. MR 20:2373

[MWh] S. Mac Lane, J. H. C. Whitehead, On the 3-type of a complex, Proc. Nat. Acad. Sci. U.S.A., 36 (1950), 41-48. MR 11:450h

[Ma] A. I. Malcev, On isomorphic representations of infinite groups by matrices, Mat. Sb. 8 (1940), 405-422.

[MS] S. Mardeśič, J. Segal, Shape Theory. The Inverse System Approach, North-Holland Math. Library vol. 26, Amsterdam, 1982. MR 84b:55020

$[\mathrm{Pa}]$ D. S. Passman, Idempotents in group rings, Proc. Amer. Math. Soc., (2) 28 (1971), 371-374. MR 44:334

[R] D. J. S. Robinson, A Course in the Theory of Groups, Springer-Verlag, 1982. MR 84k:20001

[Wa] C. T. C. Wall, Finiteness conditions for CW-complexes, Ann. of Math. 81 (1965), 56-69. MR 30:1515 
[Wd] F. Waldhausen, Whitehead groups of generalized free products, Ann. of Math. 108 (1978) 135-256.

[Wh1] J. H. C. Whitehead, Combinatorial homotopy I (and II), Bull. Amer. Math. Soc. 55 (1949), 213-245 (453-496).

[Wh2] J. H. C. Whitehead, Simple homotopy types, Amer. J. Math. 72 (1952), 1-57. MR 11:735c

Institute of Mathematics, Polish Academy of Sciences, ul. Śniadeckich 8, 00-950 WARSAW, POLAND

Address for Correspondence: Ul. Jasna 8/18, 00-013 WarsaW, Poland

Current address, of employment: Department of Mathematics and Informational Sciences, Warsaw University of Technology, pl. Politechniki 1, 00-661 Warsaw, Poland

E-mail address: dkolodz@mimuw.edu.pl 